\title{
Coding Opportunity Aware Backbone Metrics for Broadcast in Wireless Networks
}

\author{
Shuai Wang ${ }^{1} \quad$ Guang Tan $^{2} \quad$ Yunhuai Liu $^{3} \quad$ Hongbo Jiang ${ }^{4} \quad{\text { Tian } \mathrm{He}^{1}}^{1}$ \\ ${ }^{1}$ Department of Computer Science and Engineering, University of Minnesota, U.S. \\ ${ }^{2}$ SIAT, Chinese Academy of Sciences, China \\ ${ }^{3}$ Third Research Institute of the Ministry of Pubic Security, China \\ ${ }^{4}$ Department of EIE, Huazhong University of Science and Technology, China \\ \{shuaiw,tianhe\}@cs.umn.edu, guang.tan@ siat.ac.cn, \{yunhuai.liu, hongbojiang2004\}@ gmail.com
}

\begin{abstract}
Reducing transmission redundancy is key to the efficiency of wireless network broadcast. A standard technique to achieve this is to create a network backbone consisting of a subset of nodes that are responsible for data forwarding, while other nodes act as passive receivers. On top of this, network coding (NC) is often used to further reduce unnecessary transmissions. The main problem with this backbone+NC approach is that the backbone construction process is blind of what is needed by $\mathrm{NC}$, thus may produce a structure with little benefit to the NC algorithms. To address this problem, we propose a Coding Opportunity Aware Backbone (COAB) construction scheme, which seeks to maximally exploit coding opportunities when selecting backbone forwarders. We show that the better informed backbone construction process leads to significantly increased coding frequency, at minimal cost of localized information exchange. The highlight of our work is COAB's broad applicability and effectiveness. We integrate $\mathrm{COAB}$ with ten state-of-the-art broadcast algorithms, specified in eight publications [1]-[8], and evaluate it with prototype implementations with $30 \mathrm{MICAz}$ nodes. The experimental results show that our design outperforms the existing schemes substantially.
\end{abstract}

\section{INTRODUCTION}

Reducing transmission redundancy is key to the energy efficiency of broadcast in wireless ad hoc networks. Existing optimization schemes (e.g., [1]-[9]) can be divided into two categories: probabilistic and deterministic. In the probabilistic approach [9], each node rebroadcasts the packet to its neighbors with a given forwarding probability. In contrast, deterministic approach predetermines particular nodes that forward the broadcast packet. In this method, a virtual network backbone is created. Nodes on the backbone are called the forwarders, which take the responsibility of delivering packets to their neighbors, while other nodes act as passive receivers. The backbone can be constructed with tree based method [3], cluster based method [1], [7], [8], and pruning based method [2], [4]-[6].

On top of the network backbone, network coding (NC) techniques can be used to further reduce unnecessary transmissions. Originally proposed by R. Ahlswede et al. [10], this technique has been adapted to support broadcast applications in wireless networks [11]-[16]. In these work, two coding strategies, i.e., COPE type network coding (XOR) [17] and random linear network coding (RLNC) [18], are used.
XOR coding strategy is used to apply to the deterministic approach [13], [16], while RLNC is usually considered upon the probabilistic approach [11], [12].

In this paper, we consider the combination of $\mathrm{NC}$ with the deterministic approach. The main problem with traditional designs is that the backbone construction process is independent of $\mathrm{NC}$, implying that it is unaware of what is needed by NC. This may lead to a network structure of which $\mathrm{NC}$ can take little advantage. It is known that the power of $\mathrm{NC}$ depends on how many coding opportunities exist in the network [19], which is a function of packet reception status at the nodes. If such status information can be used by the backbone construction algorithm in such a way that the coding opportunities are maximized, then we can hopefully obtain more benefit from NC.

COAB contains a novel forwarder selection method to choose which nodes should broadcast packets. At the heart of this method is a metric called the per-link covering cost, which considers not only link quality, but also the reception status of neighbors. Thus we can estimate the coding opportunity and measure the broadcast efficiency of each link with $\mathrm{NC}$ in advance. With the help of the metric, COAB does not select forwarders until it calculates the best forwarding structure under current reception status. This deferred choice gives each broadcast packet multiple opportunities to make progress. As a result, $\mathrm{COAB}$ finds more opportunities for $\mathrm{NC}$ to save transmissions.

The major contribution of our work is COAB's broad applicability and effectiveness. The forwarder selection strategy can be easily combined with existing backbone construction algorithms to make the broadcast more efficient. We augment ten backbone construction algorithms, i.e., (i) tree based method [3], (ii) cluster based method [1], [7], [8], and (iii) pruning based method [2], [4]-[6], with our design. We evaluate the energy efficiency of COAB with prototype implementations with $30 \mathrm{MICAz}$ nodes. Experimental results show that compared to the traditional backbone schemes, COAB saves up to $50 \%$ of the broadcast transmissions. Our algorithm increases the coding opportunities by up to $50 \%$ compared to the backbone+NC schemes, resulting in an additional energy gain of $20-30 \%$ for typical network settings. 


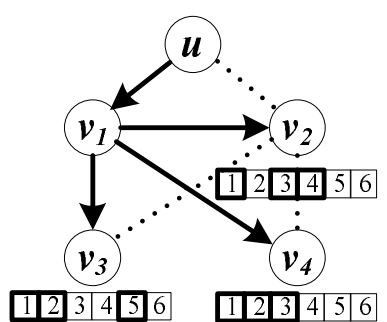

(a) Case 1: $v_{1}$ is the forwarder

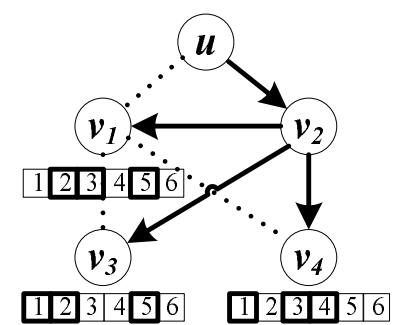

(b) Case 2: $v_{2}$ is the forwarder
Fig. 1. Impact of coding opportunity on broadcast.In the packet reception bitmap, a block with a thick borderline means received packets, and a block with a thin borderline means a lost one.

The rest of the paper is structured as follows. Section II presents the motivation of our design. Section III analyzes the model. In Section IV, we introduce the main design of COAB as well as how to integrate $\mathrm{COAB}$ with previous broadcast algorithms. Evaluation results from testbed experiments are shown in Sections V. Finally, Section VI concludes the paper.

\section{Motivation}

\section{A. Network Coding Based Broadcast Rule}

Network coding has great potential to improve the broadcast efficiency by saving redundant transmissions in wireless networks. When a source node broadcasts a coded packet to all its receivers, we need to make sure that all the receivers have already gathered enough packets to decode the new one. We specify the broadcast coding rule as follows:

Definition 1: (Broadcast Coding Rule) Consider a node $u$ transmitting an encoded packet $p^{\prime}=\oplus\left(p_{1}, p_{2}, \ldots, p_{K}\right)$. In order to decode $p^{\prime}$, each receiver should have already received $K-1$ packets among $p_{i}, i=1,2, \ldots, K$.

For NC based broadcast, we seek to encode as many packets as possible. To transmit, a node picks the first packet in its output queue, checks whether the remaining packets satisfy the broadcast coding rule and encodes as many packets as possible. Normally the number of packets that can be encoded into a single packet is small (bounded by the node's degree). Therefore, the computational overhead is insignificant.

\section{B. Coding Opportunity in Broadcast}

We use an example to show how the coding opportunity affects the efficiency of broadcast. Figure 1 shows two broadcast routes in a network, where source node $u$ wants to broadcast packets to the other nodes. In Figure 1(a), after $u$ sends the packet, node $v_{1}$ is selected as the forwarder, and node $v_{2}, v_{3}$ and $v_{4}$ are covered by the forwarder $v_{1}$. Node $v_{1}$ broadcasts the received packet (from source node $u$ ) to all the nodes it covers to accomplish the broadcast task. In Figure 1(b), similarly, node $v_{2}$ is selected as the forwarder. The broadcast task completes when node $v_{2}$ successfully delivers the packet to its covered nodes.

A node's packet reception information can be found from the packet reception bitmaps under each node in Figures 1(a) and 1(b), where a block with a thick borderline means a packet being received, and a block with a thin borderline means a
TABLE I

NOTATION USED IN THIS PAPER

\begin{tabular}{|l|l|}
\hline Notation & Description \\
\hline$e_{j}(u)=\left\{u, v_{j}\right\}$ & $\begin{array}{l}\text { A link from node } u \text { to } v_{j} \text {, we use } e_{j} \text { for short } \\
\text { when } u \text { is clear from the context }\end{array}$ \\
\hline$p(e)$ & $\begin{array}{l}\text { The link quality, measured by the transmission } \\
\text { success rate }\end{array}$ \\
\hline$\varepsilon(u)$ & $\begin{array}{l}\text { The expected transmission count for } u \text { to } \\
\text { reliable broadcast one packet }\end{array}$ \\
\hline$\beta_{n c}(u)$ & $\begin{array}{l}\text { The total number of reduced broadcast packets } \\
\text { on node } u \text { with NC }\end{array}$ \\
\hline$\xi_{n c}(V(u))$ & $\begin{array}{l}\text { The per-link covering cost of } u \text { to broadcast a } \\
\text { packet to the node set } V(u) \text { with NC }\end{array}$ \\
\hline
\end{tabular}

packet being missed. Now let's examine the number of packet transmissions needed for the two cases separately.

- CASE 1 (Figure 1(a)): Node $v_{1}$ is selected as the forwarder and it needs to retransmit packets $\left\{p_{2}, p_{3}, p_{4}, p_{5}, p_{6}\right\}$. With the help of NC, $v_{1}$ needs to retransmit packet $\left\{p_{2} \oplus p_{3}, p_{4}, p_{5}, p_{6}\right\}$ to make sure all the nodes it covers receive all the packets.

- CASE 2 (Figure 1(b)): Node $v_{2}$ is selected as the forwarder and it needs to retransmit all the six packets. With the help of NC, $v_{2}$ only needs to retransmit three packets $\left\{p_{1} \oplus p_{2} \oplus\right.$ $\left.p_{3}, p_{4} \oplus p_{5}, p_{6}\right\}$ to make up the losses on $v_{1}, v_{3}$ and $v_{4}$.

Comparing the two cases, we can see that the broadcast in CASE 2 has more coding opportunities than in CASE 1: CASE 2 has two coding operations where the first XORed packet involves 3 original packets and the second involves 2. CASE 1 only has one coding operation with 2 original packets XORed together. The total number of retransmissions for CASE 1 is 4 while that for CASE 2 is 3 . This suggests that in broadcast, if we can manage to increase the coding opportunities when we select the forwarder, then the number of transmissions can be reduced.

\section{MODEL ANALYSiS}

We aim to fully exploit NC opportunities to reduce transmissions. A basic question is: How much benefit can we get from NC? To answer the question, we first calculate the expected number of transmissions needed for reliable delivery of a packet from a source to all its receivers without considering NC. Then, we quantify the benefit of coding opportunities in reducing transmissions. Some notations used in this paper are listed in Table I.

\section{A. Expected Transmission Count}

Denote by $\varepsilon(u)$ the expected number of transmissions needed by forwarder $u$ to deliver one packet to all its covered nodes without considering NC. The total number of transmissions for the broadcast is thus $\varepsilon=\sum \varepsilon(u)$. Let the set of nodes covered by forwarder $u$ be $V(u)=\left\{v_{1}, v_{2}, \ldots, v_{M}\right\}$, where $M=|V(u)|$. Let the link quality between $u$ and its covered node $v_{j}$ be $p\left(e_{j}\right), j=1,2, \ldots, M$. The corresponding packet loss probability is denoted $p\left(\overline{e_{j}}\right)=1-p\left(e_{j}\right)$. Without loss of generality, we assume $p\left(e_{1}\right) \geq p\left(e_{2}\right) \geq p\left(e_{3}\right) \geq \ldots \geq p\left(e_{M}\right)$.

Consider the $M$ covered nodes case, where node $u$ is the forwarder and node set $\left\{v_{1}, v_{2}, \ldots, v_{M}\right\}$ is covered by $u$. 
$p\left(e_{1} \cap e_{2} \ldots \cap e_{M}\right)$ is the probability that all the $M$ receivers successfully receive a packet. Without correlated shadowing and severe interference [20], wireless links are considered to be independent [21]. This means $p\left(e_{1} \cap e_{2} \ldots \cap e_{M}\right)=$ $p\left(e_{1}\right) p\left(e_{2}\right) \ldots p\left(e_{M}\right)$.

Let $\operatorname{Pr}(\varepsilon(u)>k)$ be the probability that $u$ needs more than $k$ transmissions to deliver a packet to all the $M$ receivers, then the expected transmission count for $u$ to reliable broadcast one packet can be calculated as

$$
\begin{aligned}
E[\varepsilon(u)]= & \sum_{k=1}^{+\infty} k \cdot \operatorname{Pr}(\varepsilon(u)=k) \\
= & \sum_{k=1}^{+\infty} k \cdot(\operatorname{Pr}(\varepsilon(u)>k-1)-\operatorname{Pr}(\varepsilon(u)>k)) \\
= & \operatorname{Pr}(\varepsilon(u)>0)-\operatorname{Pr}(\varepsilon(u)>1)+2 \operatorname{Pr}(\varepsilon(u)>1) \\
& -2 \operatorname{Pr}(\varepsilon(u)>2)+3 \operatorname{Pr}(\varepsilon(u)>3)-\ldots \\
& =\sum_{k=0}^{+\infty} \operatorname{Pr}(\varepsilon(u)>k)
\end{aligned}
$$

where $\operatorname{Pr}(\varepsilon(u)>k)$ is given by

$$
\begin{aligned}
\operatorname{Pr}(\varepsilon(u)>k)= & \sum_{i=1}^{M} p\left(\overline{e_{i}}\right)^{k}-\sum_{1 \leq i<j \leq M}\left(p\left(\overline{e_{i}}\right) p\left(\overline{e_{j}}\right)\right)^{k} \\
& +\sum_{1 \leq i<j<l \leq M}\left(p\left(\overline{e_{i}}\right) p\left(\overline{e_{j}}\right) p\left(\overline{e_{l}}\right)\right)^{k}+\ldots \\
& +(-1)^{M}\left(p\left(\overline{e_{1}}\right) p\left(\overline{e_{2}}\right) \ldots p\left(e_{M}\right)\right)^{k} .
\end{aligned}
$$

Based on Eq.(1) and Eq.(2), we get the expectation for source node $u$ to reliable broadcast one packet to its covered node set $\left\{v_{1}, v_{2}, \ldots, v_{M}\right\}$.

\section{B. Coding Opportunities Estimation}

From the example in Section II, we can find that the coding opportunity is crucially dependent on the forwarder selection: we can get more benefit from $\mathrm{NC}$ if node $v_{2}$ (Figure 1(b)) is selected as the forwarder. Therefore, it is imperative to estimate the benefit of NC for each forwarder candidate. First, let's give the definition of coding opportunity:

Definition 2: (Coding Opportunity) For packets buffered in an output queue, if there exist a group of packets that satisfy the broadcast coding rule and thus can be encoded together, we call this condition a coding opportunity.

Let the number of coding opportunities with $k_{i}$ original packets involved in an encoded packet be $t_{i}, 2 \leq k_{i} \leq M$. Node $u$ 's total reduced number of broadcast packets by using network coding $\beta_{n c}(u)$ is given by

$$
\beta_{n c}(u)=\sum_{i=2}^{M}\left(k_{i}-1\right) t_{i}
$$

Note that each broadcast packet may need multiple retransmissions to ensure it being received by all the receivers. This makes great room for NC to reduce transmissions.

\section{COAB METRIC}

This section describes the main design of the COAB metric. Then we introduce how to integrate $\mathrm{COAB}$ metric with backbones construction.

\section{A. Forwarder Selection}

Consider a node $u$ with covered node set $V(u)=$ $\left\{v_{1}, v_{2}, \ldots v_{M}\right\}$. For each link $e_{j}$, the expected number of transmissions needed to successfully send a packet to node $v_{j}$ is $\frac{1}{p\left(e_{j}\right)}$. For the lost packet, we adopt a hop-by-hop retransmission model - more specifically a simple automatic repeat request (ARQ) mechanism at the MAC layer. The ARQ mechanism uses ACKs and timeouts to achieve reliable packet transmissions. If a forwarder does not receive an ACK before the timeout, it retransmits the packet until it receives an ACK or exceeds a predefined number of transmissions.

Our goal is to design a broadcast scheme that minimizes the total number of transmissions in a network using NC. Based on the observations that packet delivery efficiency highly depends on link quality and NC opportunities, we use a metric called the per-link covering cost to guide forwarder selection.

1) Impact of link status: In COAB, we define the per-link covering cost without $N C$ as follows.

Definition 3: (Per-link Covering Cost without NC) The forwarder node $u$ 's per-link covering cost is the number of transmissions needed by $u$ to deliver a packet to all of its covered nodes without using $\mathrm{NC}$, divided by the number of $u$ 's covered nodes, that is,

$$
\xi(V(u))=\frac{\varepsilon(u)}{M},
$$

where $M$ is the number of $u$ 's covered nodes. $\xi(V(u))$ offers a good estimate for the expected transmission count for a successful packet delivery without NC. It captures a basic characteristic of lossy links. $\xi(V(u))$ suggests that selecting a proper forwarder should consider covered nodes with good link qualities.

To calculate $\xi(V(u))$, we need to know $p\left(e_{j}\right)$. In wireless networks, link quality is known to be dynamic. In COAB, every node periodically sends out a HELLO message at an adaptive time interval which is increased or decreased based on the link's stability. Every HELLO message is identified by the node ID and a packet sequence number. The message is used not only for one-hop neighbor discovery, but also for updating $p\left(e_{j}\right)$. The calculation of link quality is straightforward. Every node maintains a reception record of all HELLO messages from its neighboring nodes within a time window $W$ (e.g., $\mathrm{W}=$ $6)$. In order to reduce the required memory space and mitigate the overhead of control messages, the record is represented in a bitmap format (e.g., [110010]) for each neighbor. Such records are exchanged within a HELLO message every $W$ seconds among neighboring nodes.

2) Impact of network coding: We use $\xi_{n c}(V(u))$ to denote the per-link covering cost with $\mathrm{NC}$ and call it per-link covering cost for short. 
TABLE II

TEN STATE-OF-ART PROTOCOLS SUPPORTED By COAB

\begin{tabular}{|l|c|c|c|c|c|}
\hline Protocol Name & Reference & Network Info. & Hello Msg & Broadcast Msg & Category \\
\hline Spanning Tree & {$[3]$} & One-hop & ID & Msg only & Tree-based \\
Cluster Tree & {$[1]$} & Quazi-Global & Global & Msg only & Tree and Cluster-based \\
Forwarding Node Cluster & {$[8]$} & Local & ID & Covered set & Tree and Cluster-based \\
Clustering & {$[7]$} & Quazi-Local & Degree & Msg only & Cluster-based \\
Multi-Point Relay & {$[6]$} & Two-hop & One-hop & Msg + Covered set & Pruning-based \\
Self Pruning & {$[4]$} & One-hop & One-hop & Msg + Covered set & Pruning-based \\
Partial Dominating Pruning & {$[5]$} & Two-hop & One-hop & Msg + Covered set & Pruning-based \\
Dominating Pruning & {$[4]$} & Two-hop & One-hop & Msg + Covered set & Pruning-based \\
Total Dominating Pruning & {$[5]$} & Two-hop & One-hop & Msg + Covered set & Pruning-based \\
RNG Relay Subset & {$[2]$} & Two-hop & One-hop & Msg only & Pruning-based \\
\hline
\end{tabular}

Definition 4: (Per-link Covering Cost) $\xi_{n c}(V(u))$ equals the average number of transmissions needed by forwarder $u$ to reliably deliver a single packet over a single link with $\mathrm{NC}$, that is,

$$
\xi_{n c}(V(u))=\frac{\left(|\Phi(u)|-\beta_{n c}(u)\right)}{|\Phi(u)|} \xi(V(u)),
$$

where $\Phi(u)$ is the packet set in node $u$ 's output queue. In $\mathrm{COAB}, \xi_{n c}(V(u))$ is used as the metric for forwarder selection.

\section{B. Integrating COAB Metric with Backbones}

We classify the existing reliable broadcast algorithms into tree-based [3], cluster-based [1], [7], [8], and pruningbased [2], [4]-[6]. Thus far, we have successfully implemented ten classical algorithms and embedded $\mathrm{COAB}$ with them. The basic information of these algorithms is shown in Table II. We briefly introduce how to embed our design into these tree backbone construction algorithms, and thus bringing them an improvement on energy efficiency. In Tree+COAB, instead to find the nodes with maximum leaves, we choose the nodes with $\min \left(\xi_{n c}\right)$ as the tree nodes. To combine cluster based broadcast with $\mathrm{COAB}$, the algorithm Cluster+COAB first selects nodes with $\min \left(\xi_{n c}\right)$ to form a maximal independent set (MIS). Then, Cluster+COAB finds connectors to link the nodes in MIS. In Pruning+COAB, each forwarder adds its one-hop neighbors with $\min \left(\xi_{n c}\right)$ to forwarder set to cover its two-hop neighbors.

Running the $\mathrm{COAB}$ algorithm introduces little additional communication cost. The main overhead is from two sources. One is packet reception bitmap exchange between neighboring nodes which is used to calculate the expected transmission count, coding opportunity and the broadcast link cost. The exchange of bitmap is already required by previous network coding schemes [17]. Besides, the bitmap is designed to be very short (e.g., 2 bytes) so this overhead is negligible. The other part of overhead is the exchange of one-hop neighbor information, which is required by backbone construction algorithms [5], [6], [8]. Thus, applying COAB will hardly affect the system's overall performance.

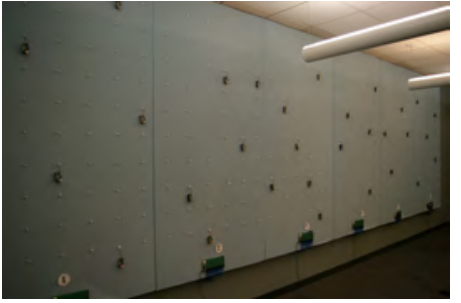

Fig. 2. Testbed

\section{TESTBED IMPLEMENTATION}

\section{A. Experiment Setup}

We deploy 30 MICAz nodes randomly on an in-door testbed shown in Figure 2. In the beginning of the experiment, a control node is used to remotely configure radio parameters, i.e., transmission power and channel. According to the testbed size, i.e., 24 feet by 8 feet, the power is set to be $-25 \mathrm{dBm}$. We use 802.15.4's channel 26, which is free of external interference (e.g.,WiFi). Based on these radio settings, each node broadcasts 100 HELLO packets in turn. Each packet was identified by a sequence number. The transmission rate is 5 packets/sec. All the received packets are recorded in the MICAz nodes' flash memory. When all the nodes finish broadcasting 100 packets, they send their packet reception information to a sink node which is connected to PC. We thus obtain the information required by $\mathrm{COAB}$, i.e., link qualities and packet receiving patterns, from packet reception history, and calculate the backbone for broadcast using the forwarder selection method. Then, the corresponding nodes in the testbed are selected as forwarders (the backbone). The forwarders keep on broadcasting packets until all their covered nodes receive 100 packet.

We use two metrics for performance evaluation: (i) Number of Transmissions, which is defined as the number of transmissions needed by a broadcast scheme to reliably broadcast 100 packets to the whole network. (ii) Number of Coding Operations, defined as the number of times that network coding occurs during the experiment. It is used to measure coding opportunities. 


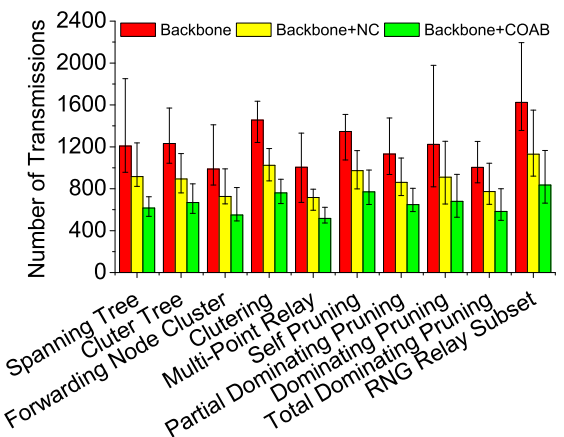

Fig. 3. Num. of Transmissions

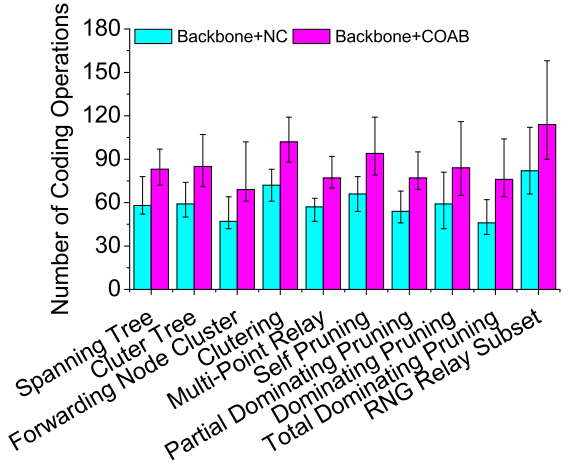

Fig. 4. Num. of Coding operations

\section{B. Main Performance Results}

The experimental results of the ten classical reliable broadcast protocols are shown in Figure 3. The first bar (in red) in each set of data represents the broadcast transmissions needed by the backbone schemes, while the second bar (in yellow) and the third bar (in green) represent the transmissions needed by backbone+NC and backbone $+\mathrm{COAB}$ schemes separately. For example, for the Spanning Tree algorithm, the nodes need 1208 transmissions on average to guarantee that every node in the network receives 100 packet, while the number is 616 when $\mathrm{COAB}$ is combined with Spanning Tree, achieving a reduction of $49 \%$. The average transmission of backbone+NC and backbone $+\mathrm{COAB}$ is 892 and 662, respectively. On average, our design $\mathrm{COAB}$ reduces transmissions of backbone+NC by $26 \%$. For the number of coding operations in Figure 4, we see that on average, backbone $+\mathrm{COAB}$ produces $43 \%$ more coding opportunities than backbone+NC. These improvements turn out to be very helpful for broadcast efficiency.

\section{CONCLUSION}

In this paper we have studied the effect of network coding opportunity on the performance of broadcast. We developed a new forwarder selection metric to capture potential coding opportunities. Our design can be widely used in broadcast algorithms. We integrate $\mathrm{COAB}$ with ten state-of-the-art broadcast algorithms, and evaluate our design with testbed experiments. The results confirm the effectiveness of our design in exploiting coding opportunities and improving energy efficiency.

\section{ACKNOWLEDGEMENT}

This work was supported in part by NSF grants CNS0917097. We also received partial support from McKnight Land-Grant Professorship.

\section{REFERENCES}

[1] K.M. Alzoubi, P.J. Wan, and O. Frieder. New distributed algorithm for connected dominating set in wireless ad hoc networks. In Hawaii Int. Conf. System Sciences, 2002.

[2] J.Cartigny, F. Ingelrest, and D. Simplot. Rng relay subset flooding protocols in mobile ad hoc networks. International Journal of Foundations of Computer Science, 2003.
[3] A. Juttner and A. Magi. Tree based broadcast in ad hoc networks. Mobile Networks and Applications, 2005.

[4] H. Lim and C. Kim. Flooding in wireless ad hoc networks. In Computer Communications Journal, 2001.

[5] W. Lou and J. Wu. On reducing broadcast redundancy in ad hoc wireless networks. IEEE Transactions on Mobile Computing, 2002.

[6] A. Qayyum, L. Viennot, and A.Laouiti. Multipoint relaying for flooding broadcast messages in mobile wireless networks. In Proceedings of HICSS, 2002.

[7] I. Stojmenovic, M. Seddigh, and J. Zunic. Dominating sets and neighbor elimination based broadcasting algorithms in wireless networks. IEEE Transactions on Parallel and Distributed Systems, 2002.

[8] J. Wu and W. Lou. Forward-node-set-based broadcast in clustered mobile ad hoc networks. In Wireless Communication and Mobile Computing, 2003.

[9] Z. Haas, J. Halpern, and L. Li. Gossip-based ad hoc routing. In Proceedings of IEEE INFOCOM, 2002.

[10] R. Ahlswede, N. Cai, S. Y. Li, and R. Yeung. Network information flow. IEEE Transactions on Information Theory, 2000.

[11] C. Fragouli, J. Widmer, and J. Boudec. A network coding approach to energy efficient broadcasting: from theory to practice. IEEE/ACM Transactions on Networking, 2008

[12] A. Asterjadhi, E. Fasolo, M. Rossi, J. Widmer, and M. Zorzi. Toward network coding-based protocols for data broadcasting in wireless ad hoc networks. IEEE Transactions on Wireless Communications, 2010.

[13] E. L. Li, R. Ramjee, M. M. Buddhikot, and S. C. Miller. Network coding-based broadcast in mobile ad-hoc networks. In Proceedings of IEEE INFOCOM, 2007.

[14] S. Chachulski, M. Jennings, S. Katti, and D. Katabi. Trading structure for randomness in wireless opportunistic routing. In Proceedings of ACM SIGCOMM, 2007.

[15] D. Koutsonikolas, C. Wang, and Y. Hu. Ccack: Efficient network coding based opportunistic routing through cumulative coded acknowledgments. In Proceedings of IEEE INFOCOM, 2010.

[16] S. Wang, A. Vasilakos, H. B. Jiang, X. Q. Ma, W. Y. Liu, K. Peng, B. Liu, and Y. Dong. Energy efficient broadcasting using network coding aware protocol in wireless ad hoc network. In Proceedings of IEEE ICC, 2011.

[17] S. Katti, H. Rahul, W. Hu, D. Katabi, M. Medard, and J. Crowcroft. Xors in the air: Practical wireless network coding. In Proceedings of ACM SIGCOMM, 2006.

[18] T. Ho, M. Medard, R. Koetter, D. R. Karger, M. Effros, J. Shi, and B. Leong. A random linear network coding approach to multicast. IEEE Transactions on Information Theory, 2006.

[19] S. Sengupta, S. Rayanchu, and S. Banerjee. Network coding-aware routing in wireless networks. IEEE Transactions on Networking, 2010

[20] K. Srinivasan, M. Jain, J. Choi, T. Azim, E. Kim, P. Levis, and B. Krishnamachari. The $\kappa$ factor: inferring protocol performance using inter-link reception correlation. In Proceedings of ACM MOBICOM, 2010.

[21] C. Reis, R. Mahajan, M. Rodrig, D. Wetherall, and J. Zahorjan Measurement-based models of delivery and interference. In Proceedings of ACM SIGCOMM, 2006. 